\title{
Point and planar LIF for velocity-concentration correlations in a jet in cross flow
}

\author{
Knud Erik Meyer, Oktay Özcan ${ }^{\dagger}$, Poul S. Larsen \\ Department of Energy Engineering, Fluid Mechanics Section \\ Technical University of Denmark, Building 403, DK-2800 Lyngby, kem@et.dtu.dk \\ ( ${ }^{\dagger}$ on leave from Department of Aero. and Astro., Tech. University of Istanbul) \\ Palle Gjelstrup, Carsten H.Westergaard \\ Dantec Measurement Technology A/S \\ Tonsbakken 16-18, DK-2740 Skovlunde, Denmark
}

\begin{abstract}
Simultaneous measurements of velocities and concentration with Planar Laser Induced Fluorescense (PLIF) combined with Particle Image Velocimetry (PIV) are compared to similar measurements with pointwise Laser Induced Fluorescense (LIF) made with a slightly modified standard Laser Doppler Anemometer (LDA). The flow considered is the mixing of a jet in a fully developed cross flow in a square duct with a width of 10 jet diameters. Both a laminar flow case, $\mathrm{Re}=675$, and a turbulent flow case, $\mathrm{Re}=33750$, are presented . For both flows, the ratio jet-to-duct mean velocities was $\mathrm{R}=3.3$. Result of mean velocities, mean concentration and Reynolds fluxes in the symmetry plane of the jet are presented for PIV and PLIF measurements. The LIF measurements performed with the LDA equipment was in general in good agreement with the PIV/PLIF measurements. The cross sections selected for comparison are challenging, since these involve areas with high velocity- and concentration gradients, which in turn amplifies the effect of a finite measurement volume in the two measurement systems. In addition, the concentration measurement was realized by injecting clean water into the dye seeded main flow. This "inverse" configuration resulted in a deeper insight to the concentration measurement process, itself. The comparison of LDA/LIF and PIV/PIF data also resulted in better understanding of the two measurement systems.
\end{abstract}

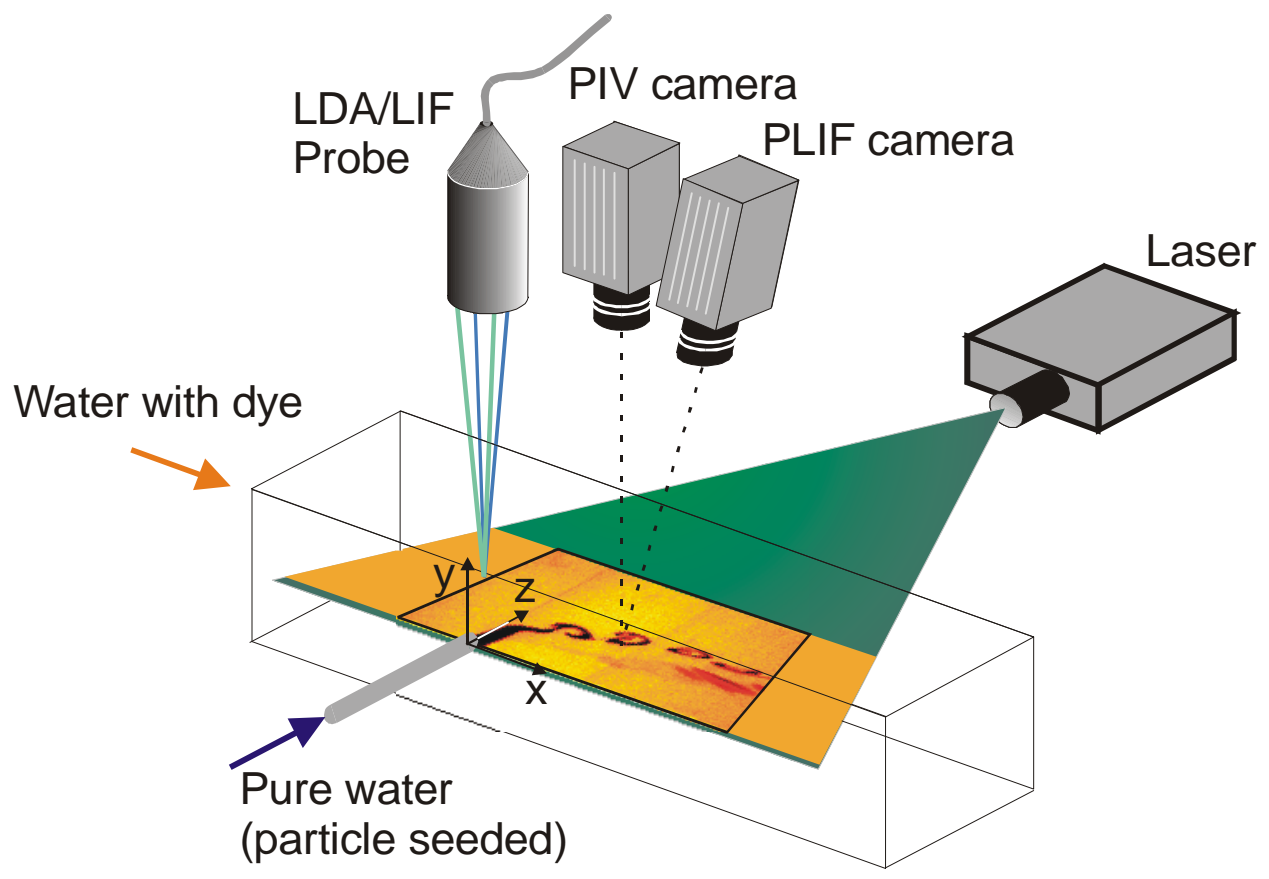

Fig. 1. Schematic diagram of the test section with the PIV and PLIF measuring system. 


\section{INTRODUCTION}

Simultaneous measurement of velocity and concentration to provide Reynolds fluxes, in addition to mean fields of concentration and velocity, is important for the understanding of mixing processes. A good way to acquire such data is to use Planar Laser Induced Fluorescense (PLIF) combined with Particle Image Velocimetry (PIV), see e.g. Houcine et al (1996) and Karasso and Mungal (1997). The present study uses the PIV/PLIF combination, but also reports data obtained by pointwise Laser Induced Fluorescense (LIF) measurements made with a slightly modified standard Laser Doppler Anemometer (LDA). Only few reports are available on this technique, e.g. Owen (1976) and Lima et al (1996). The PIV/PLIF combination has many advantages: Simultaneous measurements are easily ensured, spatial structures can be investigated and large amounts of data can be collected in a relatively short time. The LDA/LIF combination can not compete on these features but it has the advantage of high temporal resolution and it requires only simple one-sided optical access. To establish the LDA/LIF combination using an existing two- or three-component LDA system only requires an appropriate color filter for one of the photomultipliers and a coincident sampling of it's analog-to-digital converted signal.

One common way to mix two fluids in industrial processes involves jets of one fluid injected into the other fluid in a duct. While there has been many investigations of jets in a free cross flow, only few measurements have been presented for jets in a narrow duct, e.g. see Cenedese and Angelis (1998) and Camussi et al. (1998). The present study considers a single jet entering fully developed laminar and turbulent flow in a straight duct with a square cross section. The ratio between jet velocity and duct velocity was chosen so that the jet trajectory reached approximately the middle of the duct. Other jet ratios were measured but these are not reported in the paper. The purpose of the present paper is to investigate the performance of the LDA/LIF combination compared to the more conventional PIV/PLIF combination, and detailed data of velocity, concentration and Reynolds fluxes in the jet plane of symmetry are presented. For the comparison, challenging cross-sections of the flow are shown.

\section{EXPERIMENTAL METHOD}

\subsection{Test section and flow system}

The test section (see Fig. 1) consists of a straight duct with a square cross section of $40 \times 40$ mm manufactured of 10 mm thick Perspex plate. A glue smear in the duct corners made optical access difficult in a region of 1-2 mm from the corners. The jet, emerging from the mid-point of one side wall and perpendicular to this wall, is created from a pipe with an inner diameter of $\mathrm{D}=4 \mathrm{~mm}$ and a length of $50 \mathrm{D}$. The jet consists of clean water. The water comes from a separate reservoir through a pump, a manual control valve and a flow meter. The upstream length of the duct is 63 hydraulic diameters. The inlet to the duct is fitted with a flow straightener consisting of 4 by 4 tubes, of 9 mm inside diameter, $10 \mathrm{~mm}$ outside diameter, and $200 \mathrm{~mm}$ in length. The duct is placed in a recirculating water loop consisting of a 250 liters water reservoir, a centrifugal pump, a manual control valve and an electromagnetic flow meter. The water temperature was $26^{\circ} \mathrm{C}$. Fluorescent dye (Rhodamine 6G) was added to the water reservoir before each measurement series. The jet flow was pure water and an overflow device ensured that the water inflow from the jet did not change the water level in the water reservoir. Both the duct and the jet water were seeded with $5 \mu \mathrm{m}$ polystyrene particles.

Two flow rates have been investigated: a laminar flow with $\operatorname{Re}=675$ and a turbulent flow with $\operatorname{Re}=33750$. The Reynolds number $R e$ is based on hydraulic diameter and bulk mean velocity $U_{b c}$ of the duct flow. For both flows the ratio jet-to-duct mean velocities was $\mathrm{R}=3.3$. Both the duct flow and the jet pipe flow can be considered fully developed. The coordinate system is centered at the jet axis at the entrance to the duct, $\mathrm{x}$ being in the direction of duct flow and $\mathrm{z}$ in the direction of the jet axis. All mesurements are made in the $\mathrm{x}-\mathrm{z}$ plane centered in the duct, yielding the $\mathrm{U}$ and $\mathrm{W}$ components of velocity in $\mathrm{x}$ and $\mathrm{z}$, respectively.

\subsection{PIV and PLIF system}

\subsubsection{PIV/PLIF set-up and recording}

Two Dantec HiSense cameras, both mounted with a 60mm/F2.8 Nikkor lens, were used for the data recording. The cameras have a Peltier cooled 1280 by 1024 pixel CCD with a 6.7 micron pixel pitch. The camera used to record the PIV images was equipped with a 532nm narrow band green filter ensuring that only the light scattered from the $5 \mu \mathrm{m}$ polystyrene particles was captured. The PIV camera was mounted with the optical axis perpendicular to the light sheet and set at a magnification of $\mathrm{M}=6.34$. 
The PLIF camera was positioned slightly off axis (approx. 7 degrees) and was fitted with a 560nm cut-off filter to ensure the fluorescence light to be captured while adequately suppressing the 532nm laser light. The PLIF camera was operated in binning mode, which means that 2 by 2 pixels is integrated in the camera head. This mode makes the recording more sensitive. The fluorescent signal was quite strong, and the binning mode was mainly chosen in order to reduce the amount of data and to reduce pixel noise.

The light sheet was generated by a double cavity $120 \mathrm{~mJ}$ New Wave Nd:Yag laser with a light sheet of the size $300 \mathrm{x} 2$ $\mathrm{mm}$. Typical settings of the laser was between $18 \mathrm{~mJ}$ and $42 \mathrm{~mJ}$. The peak light intensity in the light sheet was quite high, 2 to $5 \mathrm{MW} / \mathrm{m}^{2}$, which is somewhat higher than advisable (Speiser \& Shakour 1985), because it produces nonlinear fluorescence in response to light intensity. However, the response in terms of fluorescent light to concentration was found still to be quasi-linear. The non-linear fluorescence to the light intensity only makes the conversion from measured emitted light to concentration in absolute terms somewhat more tedious.

\subsubsection{Co-ordinate system}

The PIV camera is used as the master coordinate system. In order to verify the origin and the magnification, a laminar duct flow was generated without a jet. By fitting the flow profile of the duct with a parabola, both the magnification and the position of the walls was accurately determined, defined by $\mathrm{U}(\mathrm{z})=0$, although the wall was not seen in the image. The laminar duct flow case also verified that the camera pixel co-ordinate system was aligned with the duct wall within one pixel over the entire CCD with the side-length of 1280 pixel. The determination of the co-ordinate system origin in the flow direction was achieved by locating each side of the jet pipe inside the Perspex wall of the duct. The $4 \mathrm{~mm}$ jet diameter is represented by 94 pixels. The entire image covered the $40 \mathrm{~mm}$ duct width from $\mathrm{x}=-17 \mathrm{~mm}$ upstream to $\mathrm{x}=37$ mm downstream of the jet exit.

In order to define the co-ordinate system in the PLIF camera, a set of single light pulse exposed images was recorded simultaneously with both the PIV and PLIF camera. The PLIF camera was temporally fitted with a green filter, identical to the one on the PIV camera, allowing particle images to be recorded. By using a standard cross-correlation between the two cameras, co-ordinate transformation from the PIV camera to the PLIF camera was determined. The co-ordinate transformation was fitted with a function $(i, j)=p(x, z)$, where $p$ is a second order polynomial and $(i, j)$ is the pixel location in the PLIF camera. The resulting correction over the PLIF camera was between 0 and 54 pixels.

Using this method for coordinate transformation, the positioning accuracy between the two cameras was far better than one pixel $(<<0.05 \mathrm{~mm})$. This accuracy is important in order to ensure satisfactory Reynolds fluxes.

\subsubsection{PIV data analysis}

The timing between the two light pulses was typically gauged to give a displacement of the order of 10 pixels for the bulk main flow, corresponding to 0.1 jet pipe diameters. Using an adaptive correlation algorithm (often also referred to as multigrid, i.e., Scarano and Riethmuller, 1999) allowed capturing jet exit velocities satisfactorily. The final interrogation area was chosen to be 32 pixel square, resulting in an almost $\sim 100 \%$ validation in the main flow. This size is also in balance with the light sheet thickness of $2 \mathrm{~mm}$ considering the flow is strongly three dimensional in the vicinity of the jet exit. The 32 pixel corresponds to $1.35 \mathrm{~mm}$. $50 \%$ overlap between the interrogation areas was chosen, so the computed velocity vectors were spaced $0.67 \mathrm{~mm}$ or 0.17 jet pipe diameters.

From the initial investigations of the laminar pipe flow the uncertainty of the velocity vectors are estimated to be 0.015 $\mathrm{m} / \mathrm{s}\left(2 \% * \mathrm{U}_{\mathrm{bc}}\right)$ for the turbulent case and $0.4 \mathrm{~mm} / \mathrm{s}\left(2.4 \% * \mathrm{U}_{\mathrm{bc}}\right)$ for the laminar flow case. 1000 samples were used for the turbulent case and 500 samples were used for the laminar case. The time between samples was in both cases $1 \mathrm{~s}$. For the turbulent case this ensures statistically independent samples. For the laminar case the time between samples corresponds to the passage of the largest vortices. The number of statistically independent samples is therefore somewhat lower than the 500 samples that were actually taken for the laminar case.

\subsubsection{PLIF data analysis}

In order to establish reference PLIF images, a series of images was recorded without jet flow. A known quantity of Rhodamine 6G dye was added stepwise into the closed flow loop, and circulated to ensure satisfactory mixing and the response of the fluorescent light (grey value in the camera) was monitored to ensure linear response to the dye concentration. The maximum concentration used was $0.038 \mathrm{mg} / \mathrm{l}$, which has earlier (Ullum, 1999) been verified as within a linear response range. 
A reference image (taken with a laser pulse energy of $18 \mathrm{~mJ}$ ) for the laminar flow case was generated as an ensemble average of 10 images with the laser set at the same pulse energy as the data recording. Since in the image there is a homogeneous concentration of dye in the entire duct, this reference image contains full information needed for the correction of optical imperfections. The imperfections entail details such as: light sheet beam profile after entering the duct section, miscellaneous optical variations in the imaging system (mainly the duct wall), and light damping across the duct due to absorption by the dye.

As described above, the optical system imperfection is easily mapped in a reference image. Dying the entire duct and injecting a pure water jet, as to the opposite injecting a dye jet into a clean water main flow, has some additional benefits. One benefit is that the laser light fluctuations from image to image can now be monitored upstream of the jet simply by establishing a reference point in the middle of the duct. Since the duct flow is established in a closed water loop, injecting the pure water slowly dilutes the absolute dye concentration, which is also monitored at the upstream reference point and corrected for in each image.

Green light scattered from particles in the flow and imperfections in the Perspex wall excites the dye outside the light sheet. This means that the fluorescent light originating from outside the light sheet is also captured by the PLIF camera and generates a higher background level than what would be expected from a recording without dye in the duct. The higher background level is clearly detected at the pure water jet exit, and is used from recording to recording in defining zero dye concentration. The concentration $\mathrm{C}$ has been normalised to have the upstream reference point correspond to zero concentration and the jet reference point (with pure water) correspond to a concentration of unity, i.e. in terms of pixel levels:

$$
\mathrm{C}=(\text { local level }- \text { upstream level)/(jet level - upstream level). }
$$

The final step in obtaining a concentration map from a PLIF recording to eliminate pixel to pixel noise by averaging an area of 4 by 4 binned pixels (corresponding to an area of 8 by 8 pixels in PIV image) around the centre pixel for the corresponding PIV interrogation area. The centre pixel of the 4 by 4 area is found by the previously described coordinate transformation between the PIV and PLIF camera. The result is a spatial resolution which is a quarter of the velocity vector resolution, $0.33 \mathrm{~mm}$ or 0.085 jet pipe diameters. However, the resolution perpendicular to the light sheet is determined by the light sheet thickness of $2 \mathrm{~mm}$. In terms of the absolute concentration, the result is quite good, which can be seen by observing the concentration variation in the $\mathrm{x}$ and $\mathrm{z}$ directions for low jet ratio experiments (not included in this paper). At upstream points with uniform distribution of the dye, the RMS value of the concentration $\mathrm{C}$ is found to be $\mathrm{C}_{\mathrm{rms}}=0.025$. This indicates the accuracy one can expect. The 12-bit resolution is therefore more than adequate here.

For the turbulent case, a pulse-energy of $42 \mathrm{~mJ}$ was used. Since this energy is clearly within the non-linear response to light intensity, the previously recorded reference image can not be used. The recording of a reference image at this pulse energy was not performed, so a reference image had to be generated analytically based on data fitting to the previous reference image and ad-hoc correction for the light intensity. The result is adequate in terms of absolute concentration, however overlayed by an unphysical gradient with variation of about $15 \%$. For the Reynolds fluxes, the quality of the correction is of little or no consequence, because the average quantity is eliminated.

\subsection{LDA and LIF system}

The LDA/LIF system consisted of a standard Dantec LDA system with a two-channel FVA processor and one BSAprocessor. The FVA was used to measure two velocity components, whereas the BSA was used as a power supply and amplifier for a third photomultiplier, equipped with a colour filter allowing only fluorescent light emitted from the flouresent dye to pass. The PM output signal from the BSA processor was connected to an analog-to-digital conversion card (AD-card) in the computer used to control the FVA processor. The AD-card was triggered by the FVA's burst detector such each velocity measurement by the FVA triggered a reading of the PM output signal. The Reynold's fluxes were obtained from the coincident velocity and concentration data.

Using an optical probe that operates in back scatter mode causes a special problem near walls. The presence of a wall reduces the effective aperture of the receiving optics. This causes the LIF signal to decrease when approaching a wall even though the actual concentration is constant. This error was successfully removed by covering the one half of the front lens that was closest to a wall. To make measurements of two velocity components close to the walls a special optical probe (originally designed for three-component measurements) was used. The probe has two beam pairs in orthogonal planes. The beam pair measuring $\mathrm{W}$ velocity consists of a center beam and a beam at a radius of $37 \mathrm{~mm}$ using light at $514.5 \mathrm{~nm}$. The beam pair measuring U velocity has two beams on a diameter of $74 \mathrm{~mm}$ using light at 
$476.5 \mathrm{~nm}$. Light was supplied by an Ar-ion laser with an output of 1.8 W (all wavelengths). The outer diameter of the probe is $112 \mathrm{~mm}$. The probe expands the beams internally to $4.1 \mathrm{~mm}$ diameter. With a front lens of $240 \mathrm{~mm}$ focal length this yields measurement volume for U-velocities with diameter $0.04 \mathrm{~mm}$ and length $0.24 \mathrm{~mm}$ and a measuring volume for $\mathrm{W}$-velocities with diameter $0.04 \mathrm{~mm}$ and length $0.50 \mathrm{~mm}$. The size of the measuring volume for LIF is estimated to be about 4 times the size of the measuring volume for $\mathrm{W}$-velocities. This estimate is based on ray-tracing on the receiving optics for scattered light in different planes. The optical probe was tilted an angle of $5^{\circ}$ to avoid the region near corners with poor optical access.

The concentration was found in a similar way as used by PLIF measurements. The concentration was again normalized with upstream and jet light intensity levels corresponding to a concentration of unity in the jet core and a concentration of zero upstream of the jet. The light intensity from the reference measurement in the jet core clearly changed with the thinning of the Rhodamine dye in the same way as found by PLIF measurements. This is because the receiving optics also collects some light from fluorescence outside the focal area. This effect is compensated for by using two reference points. Each line profile was carried out as follows: first an upstream point $(x, z)=(-20 D, 5 D)$ and then a point in the jet core $(x, z)=(0,0.25 D)$ was measured to get the reference values. After measuring the line profile data, the two reference points were measured once more. This was done to obtain information of the effect of the thinning of the dye due to the jet flow into the system. The actual reference levels for each measuring point in the line profile was found by linear interpolation in time of the reference values found before and after the line profile data was taken. The measuring time in each point was $60 \mathrm{~s}$. Based on measured time autocorrelations this was found to correspond to about 700 independent samples for velocity measurements. Data rates for velocities were in the range $30-250 \mathrm{~Hz}$ with the lowest data rate found for the U-velocity component. The statistics for velocities was based on residence time averaging. The RMS value of the concentration in upstream measurements (with constant concentration of dye) was about $10 \%$. This indicates the accuracy of a single LIF measurement. The accuracy of a single velocity measurement is estimated to $2 \%$.

\section{RESULTS AND DISCUSSION}

\subsection{Upstream measurements}

In Fig. 2 upstream measurements of mean velocities are compared with results from other workers. The PIV data are measured about 4D upstream from the jet in a measurement series with a jet ratio of 1.3. The presence of the jet may explain the slight asymmetry found in the mean velocity profile. There is a $1.5 \%$ difference in absolute velocities measured by PIV and LDA, which may be the difficulty in achieving exactly the same flow in the two tests. The shape of the mean velocity profile is in good agreement with the data from numerical calculations by Choi et al (1989). The measured values of $\mathrm{U}_{\mathrm{rms}}$ and the $\overline{u w}$ Reynolds stress are compared to numerical calculations in a square duct by Gavrilakis (1992) at a low Reynolds number and to measurements of Comte-Bellot (1965) between parallel plates at a Reynolds number similar to the present measurements. Data has been normalised by the wall friction velocity with the value $\mathrm{u}_{\tau}=0.039 \mathrm{~m} / \mathrm{s}$ found from the correlation of the friction factor offered by Jones (1976). The agreement between the data sets is good. There is some scatter in the PIV and LDA data. The limited number of independent samples

probably causes this. The measured $\overline{u w}$ Reynolds stresses are in very good agreement with a line through $(0,0)$ with a slope of 1.0 (shown as a dotted line). This demonstrates good agreement with the correlation for the wall friction by Jones (1976).

\subsection{PIV and PLIF measurements}

Figs. 3 and 4 present coloured contour plots of the mean concentration $C$ for the laminar and turbulent flow cases, respectively. Time-averaged velocity vector fields measured by PIV/PLIF are superimposed on the contour plots. The white line indicates the stream trace of mean flow, which passes through the center of the jet at the exit (origin of the co-ordinate system). As noted by Yuan and Street (1998), this stream trace is the best indicator of the jet trajectory. Alternatively, we can define a scalar jet trajectory as the locus of points with maximum concentration. For $\mathrm{x} / \mathrm{D}>2$, the locations of maximum concentration fall below the stream trace jet trajectory, i.e., the scalar trajectory is located below the stream trace trajectory. This behaviour of the trajectories has also been observed by Yuan and Street (1998) who offered the following explanation: the circulation produced by the counter rotating vortex pair draws scalar concentration away from the center plane at the top of the jet cross section and returns the scalar concentration towards the centerplane at the bottom of the jet cross section. Comparison of Figs. 3 and 4 shows a broader green region 

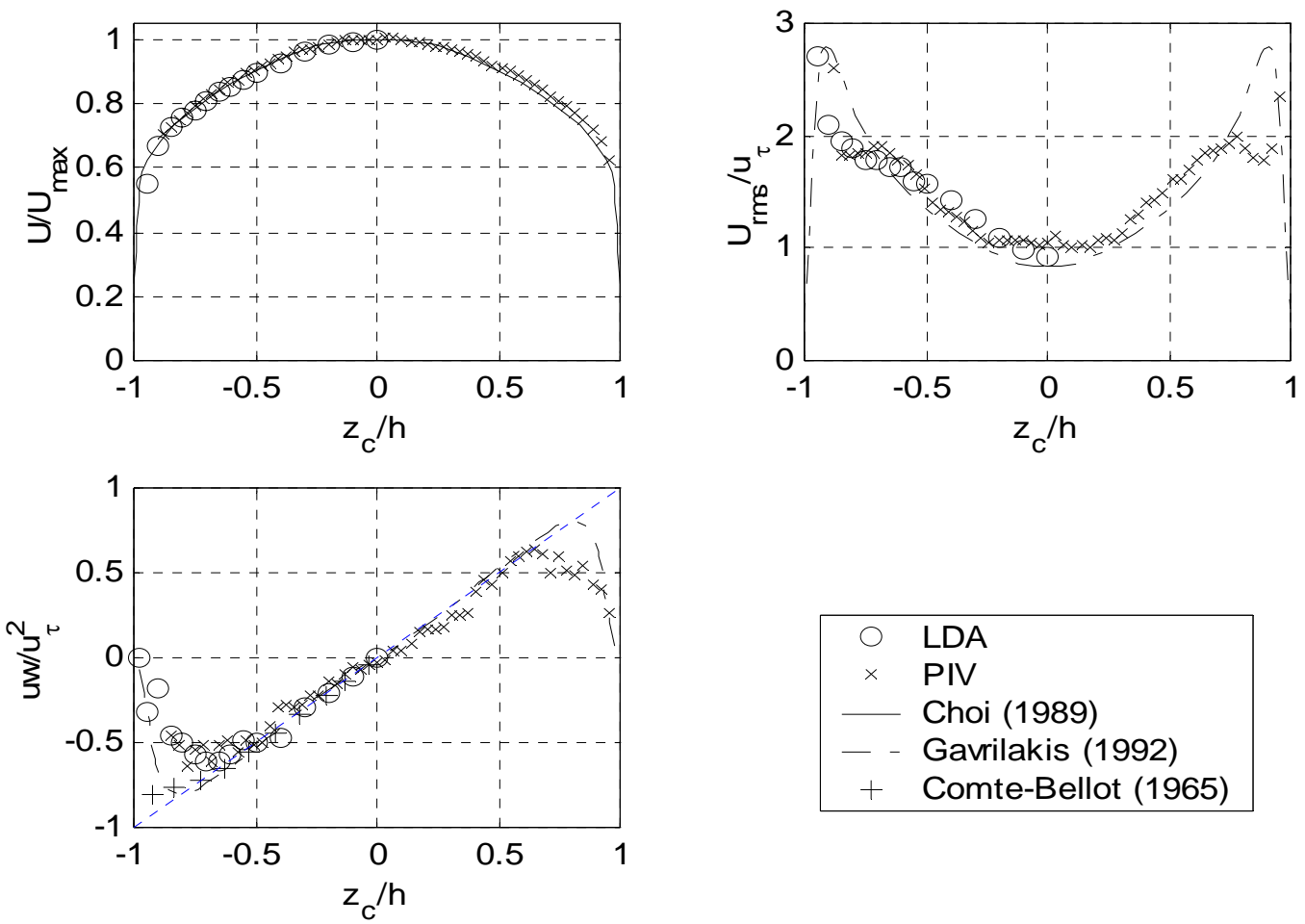

Fig. 2. Upstream profiles measured with LDA and PIV $\left(\mathrm{z}_{\mathrm{c}}\right.$ is distance from center of the duct and $\mathrm{h}$ is the half-width of the duct).

(corresponding to a concentration level around 0.5) for turbulent flow, which has a much higher mixing efficiency than the laminar flow.

Comparison of Figs. 3 and 4 also reveals that penetration of the jet into the duct is much higher for the laminar case than the turbulent one. This is due to the higher diffusion of the jet momentum and larger kinetic energy of the incoming flow for the turbulent case. Figs. 3 and 4 show that there are significant differences between the stream trace topologies of the turbulent and laminar flow cases. It is interesting to note the presence of a low momentum reverse flow region around $\mathrm{x} / \mathrm{D}=2$ and $\mathrm{z} / \mathrm{D}=3.5$ for the laminar case.

Figs. 5 and 6 present coloured contour plots of the Reynolds fluxes $\overline{u c}$ and $\overline{w c}$, respectively, superimposed on the mean velocity vector field of the turbulent flow. The magnitude of the $\overline{w c}$ flux is approximately three times larger than the $\overline{u c}$ flux. It appears that the relative magnitudes of $\overline{w c}$ and $\overline{u c}$ are proportional to the velocity ratio (which is 3.3 in this case). For $\mathrm{x} / \mathrm{D}>1$, the $\overline{w c}$ flux is always positive above the jet trajectory and generally negative below the jet trajectory. There is not such a definite trend for the $\overline{u c}$ flux, except for the fact that negative $\overline{u c}$ values are generally located above the jet trajectory.

\subsection{LDA velocities in comparison to PIV}

Figs. 7 and 8 shows some of the LDA/LIF measurements for the turbulent flow conditions. The data are plotted together with data extracted from the PIV/PLIF measurements. Mean and RMS values of velocity components are shown. The plots in Fig. 7 along the $\mathrm{x}$-direction (main flow direction) for $\mathrm{z}=3 \mathrm{D}$, show a cross section of the high 


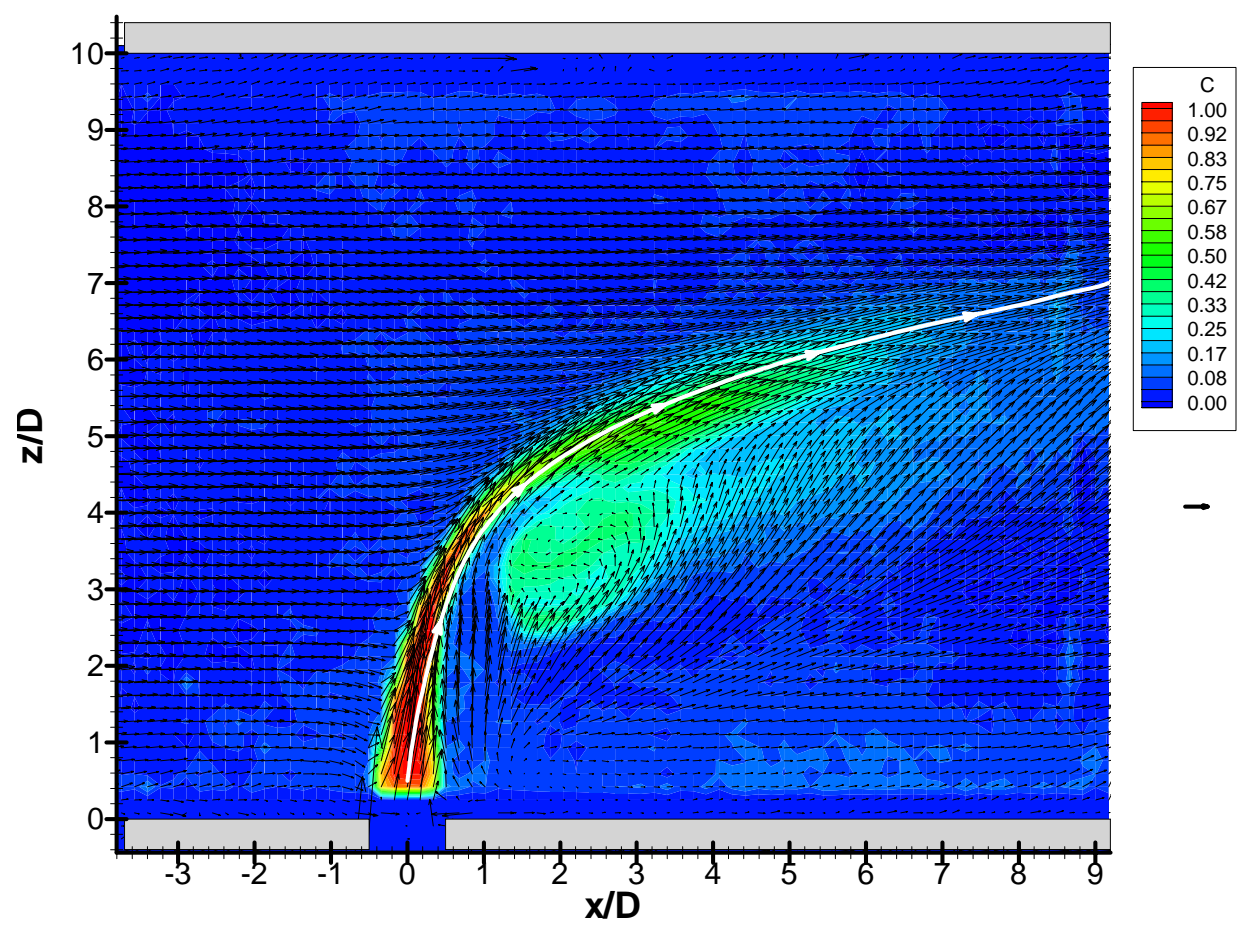

Fig. 3. Mean concentration $\mathrm{C}$ together with mean velocity vectors for laminar flow case

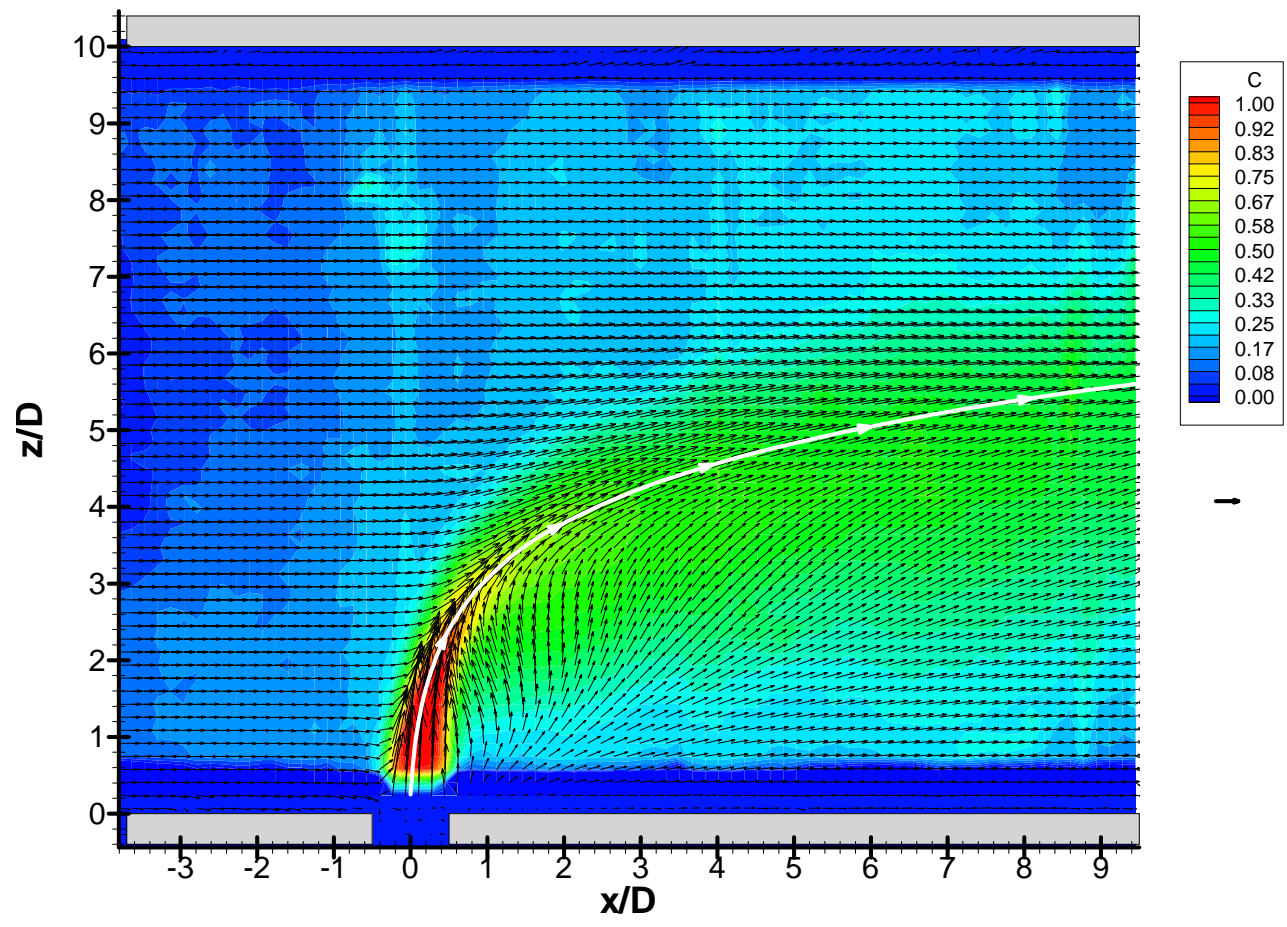

Fig. 4. Mean concentration $\mathrm{C}$ together with mean velocity vectors for turbulent flow case 


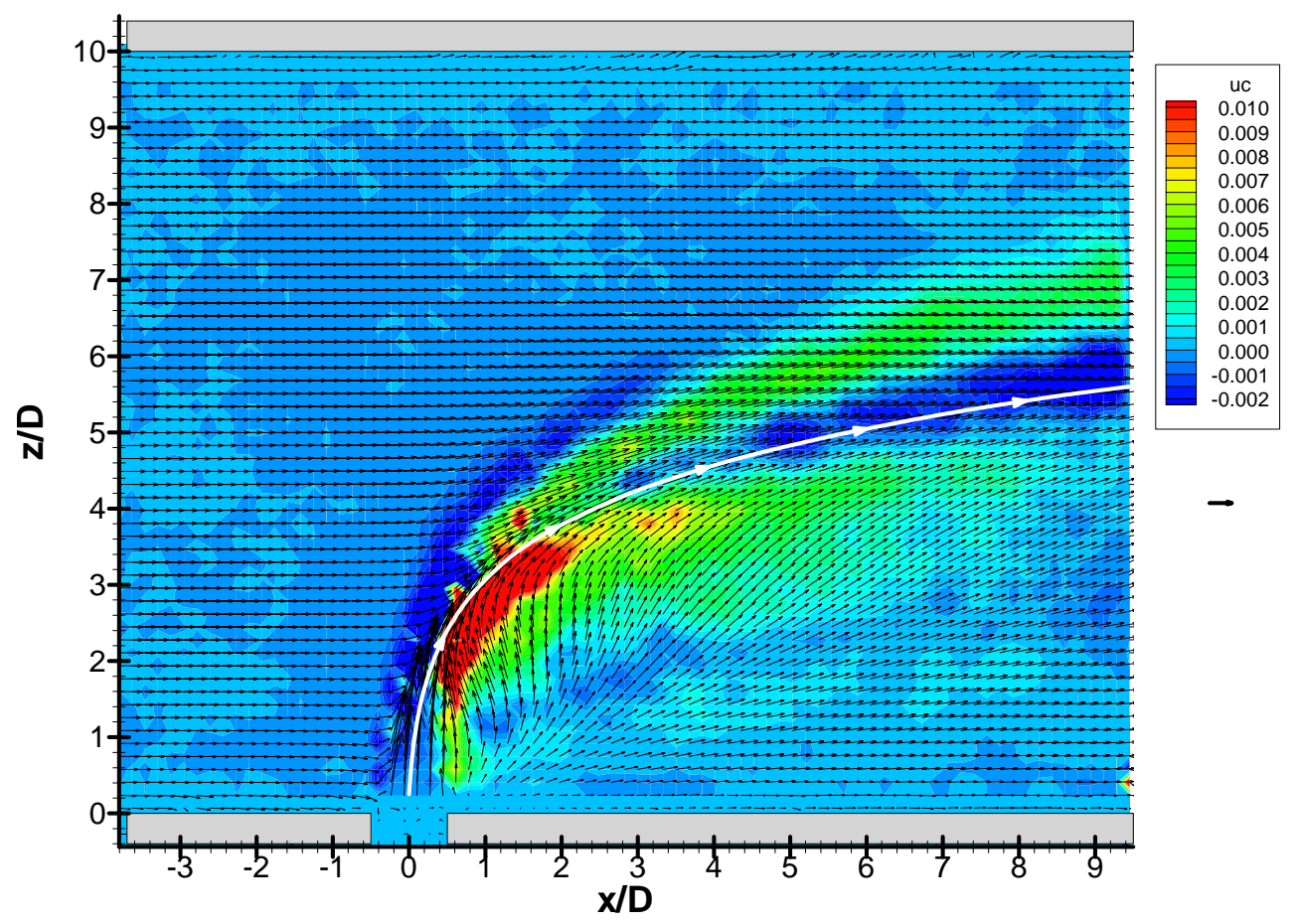

Fig. 5. The $\overline{u c}$ Reynolds flux together with mean velocity vectors for turbulent flow case.

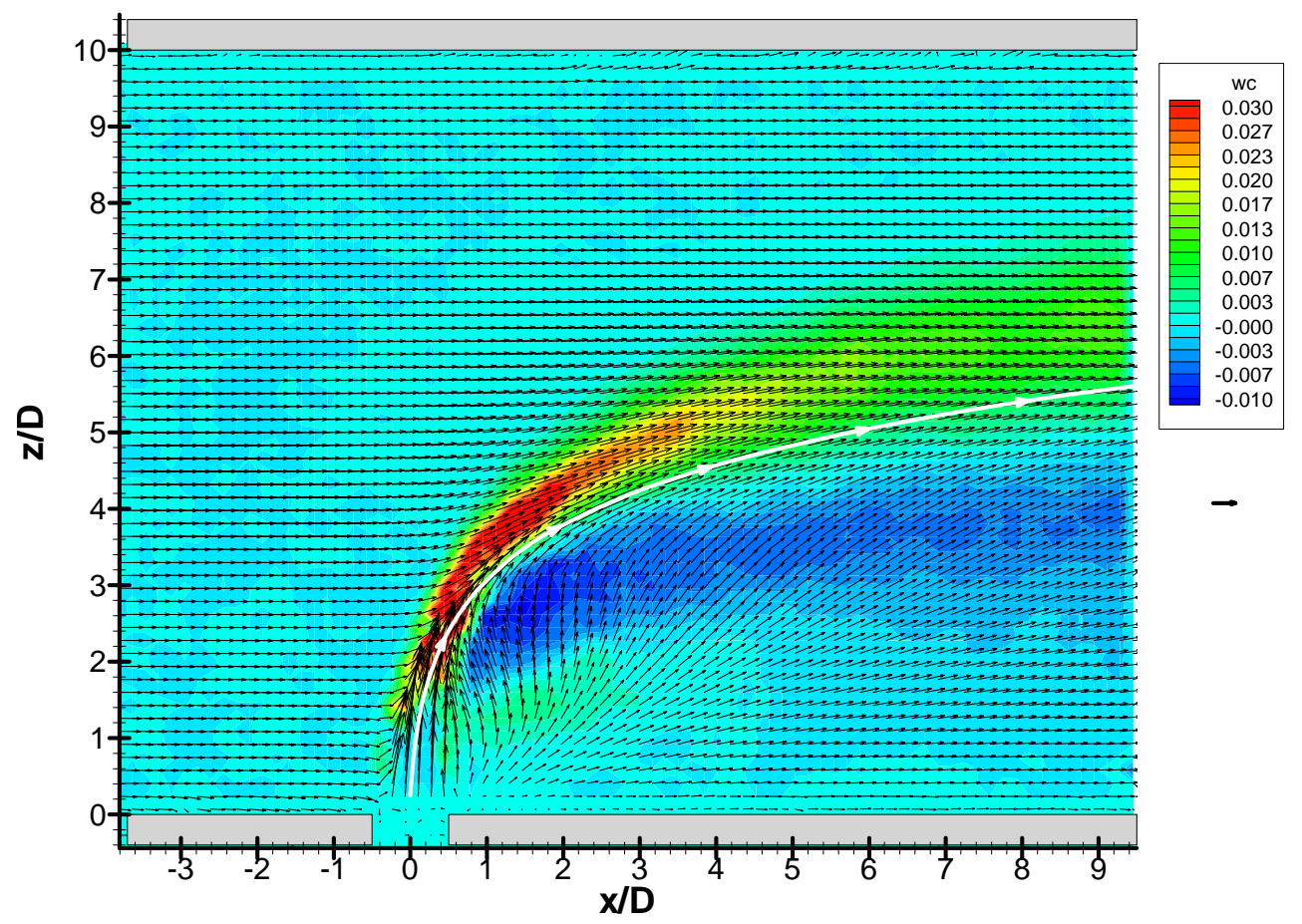

Fig. 6. The $\overline{w c}$ Reynolds flux together with mean velocity vectors for turbulent flow case. 
velocity region in the jet. The plots in Fig. 8 along the $\mathrm{z}$-direction for $\mathrm{x}=3 \mathrm{D}$ show a cross section behind the bent jet. Both cross sections are located in the most intense mixing region, in which the largest measurement deviations are also to be found.

The mean value of the U-velocity component measured with LDA is in good agreement with the PIV measurements. In both Figs. 7 and 8, differences in measured W components are found in the region near $(\mathrm{x}, \mathrm{z})=(3 \mathrm{D}, 3 \mathrm{D})$, which is about 2 jet diameters downstream of the jet trajectory. This region is a low velocity region, which is thin in the $y$-direction and would therefore not be resolved by the PIV measurements with a light sheet thickness, which is $2 \mathrm{~mm}$, or half of the jet exit diameter. The RMS-values of velocities shown in Figs. 7 and 8 are a bit higher for LDA measurements compared

to PIV. The PIV measurement volume of $1.35 \times 1.35 \times 2 \mathrm{~mm}^{3}$ (interrogation area and light sheet thickness) is significantly larger than the LDA measurement volume of only $0.04 \times 0.04 \times 0.5 \mathrm{~mm}^{3}$. Some small scale motions are probably not captured by the PIV measurements resulting in too low estimates of the RMS values.

It should again be pointed out that the two cross sections presented in Figs. 7 and 8 are the more challenging points of the flow. In addition it should be considered that small differences in flow condition and a minor co-ordinate mismatch will result in great differences comparing the $\mathrm{W}$-component at this location. For the upstream measurements presented in Fig. 2, much better agreement is found.

\subsection{LDA/LIF and PIV/PLIF concentration measurements}

As described earlier, the fluorescent signal was scaled on the basis of the up-stream signal level and the signal level in the core of the jet exit. This method was used in both the LDA/LIF and the PIV/PLIF measurements. Further, for the PIV/PLIF the scaling is based on a reference image recorded at jet-off conditions.

In Fig. 3, it is seen that the absolute concentration level is determined without any significant artificial background offset whereas in Fig. 4, a clear background gradient is seen both in an upstream profile $(x / D=-3)$ and outside the jet in the main flow direction $(\mathrm{z} / \mathrm{D}=9)$. In several regions the upstream concentration is found as $\mathrm{C} \approx 0.1$ even though it by definition should be zero. This background gradient is entirely due to the ad-hoc reference images, which were generated for the turbulent flow case, see section 2.2.4. It should be noted that the gradient only affects the absolute concentration measurement, since the gradient is averaged out in the calculation of concentration RMS and the Reynolds fluxes. For the line profiles shown in Figs. 7 and 8, the concentration has been corrected using nearby upstream points as reference.

The concentrations found by LDA/LIF and PIV/PLIF are in general in good agreement, but at some locations there are significant deviations. In Fig. 7 the PIV/PLIF measurements find a clear peak at the jet passage $(x=1 D)$ while LDA/LIF measurements find only a low peak. An explanation is that due to the higher resolution of LDA/LIF it will detect the lower concentration found near the centerline of the "kidney" shaped vortex region. The large measuring volume of PIV/PLIF is here likely to find a too large concentration. In Fig. 8 the LDA/LIF measurements of concentration show considerable scatter. To see if this was caused by noise the experiment was repeated at a slightly lower jet-to-duct velocity ratio, but results showed almost the same distribution.

The level of the RMS values of concentration is significantly higher for LDA/LIF than for PLIF measurements. This difference is ascribed to the higher optical noise level for the LDA/LIF data (about 10-15\% compared to 2.5\% for PLIF). The photomultimplier receives a small amount of scattered light originating from outside the measuring volume. This effect should increase with decreasing upstream concentration of Rhodamine, which is confirmed by comparing results in Figs. 7 and 8, noting that the upstream concentration was lower in Fig. 8 than in Fig. 7.

In Fig. 8, there is quite good agreement between the $\overline{w c}$ Reynolds flux found by the two techniques. The agreement between the measured $\overline{u c}$ Reynolds flux in Fig. 8 is acceptable in view of the quite low level of the Reynolds flux. In Fig. 7 the Reynolds fluxes measured by LDA/LIF are much higher than those measured by PIV/PLIF in the region close to the jet trajectory. This region has high mixing at scales smaller than the jet diameter and again, the large measuring volume of PIV/PLIF will lead to too low levels of the Reynolds fluxes. 

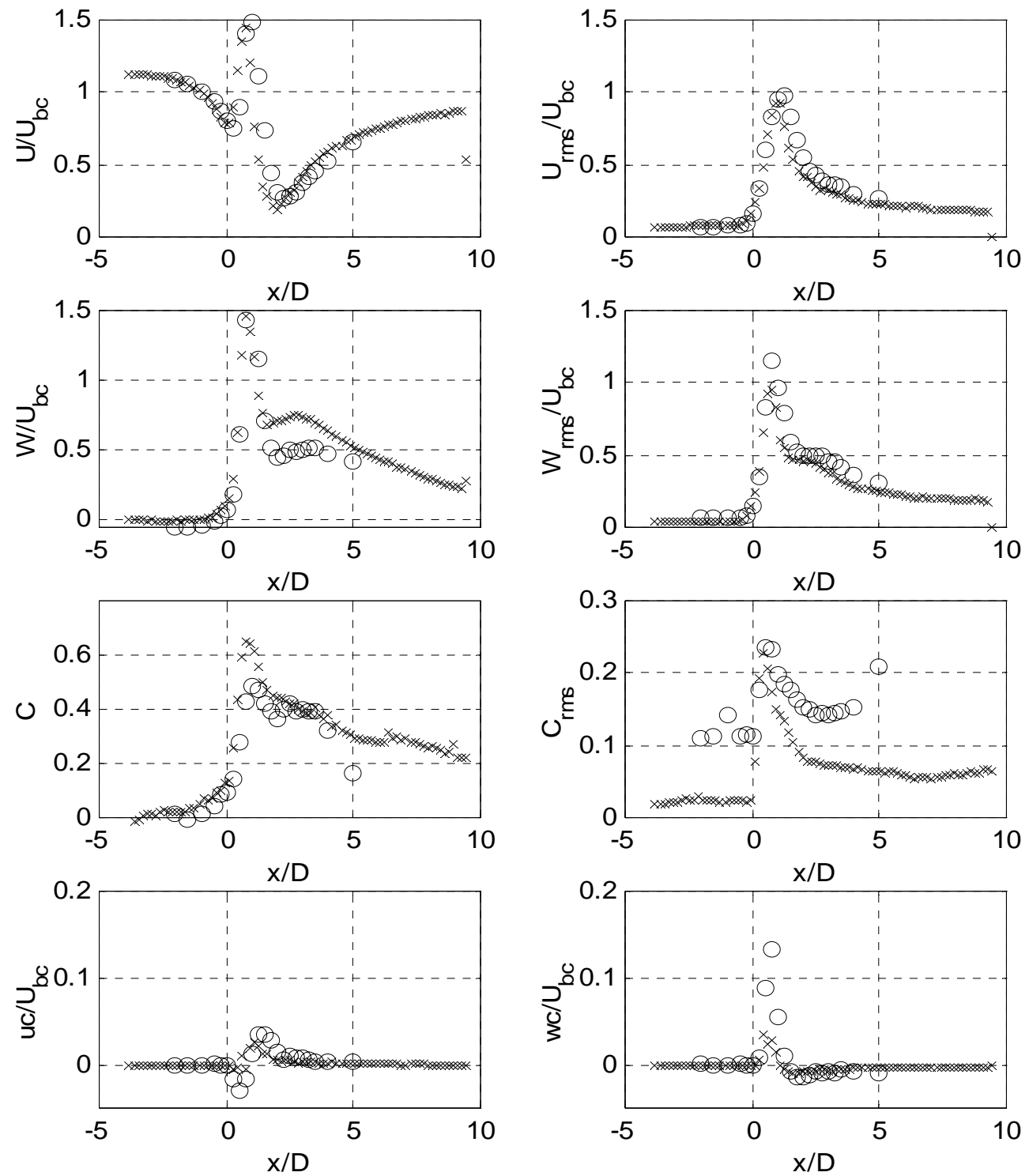

Fig. 7. Profiles of velocity components and concentration (mean and RMS values) for turbulent flow along a line through $\mathrm{z}=3 \mathrm{D}$. Symbols used in the plots: LDA/LIF: $\mathrm{O}$ and PIV/PLIF: $\times$.

The LDA/LIF measurements at points with high mixing showed rapid fluctuations in the concentration signal. Time autocorrelation of the concentration signals indicated that even with the high noise level taken into account, the time scales of the concentration fluctuations were in general significantly smaller than the corresponding time scales of velocity fluctuations. The diffusivity of Rhodamine into water is about a factor 1000 lower than the kinematic viscosity of water. This supports that scales of concentration fluctuations would be smaller than the scales of velocity fluctuations. A small measuring volume is therefore essential for obtaining accurate Reynolds fluxes. 

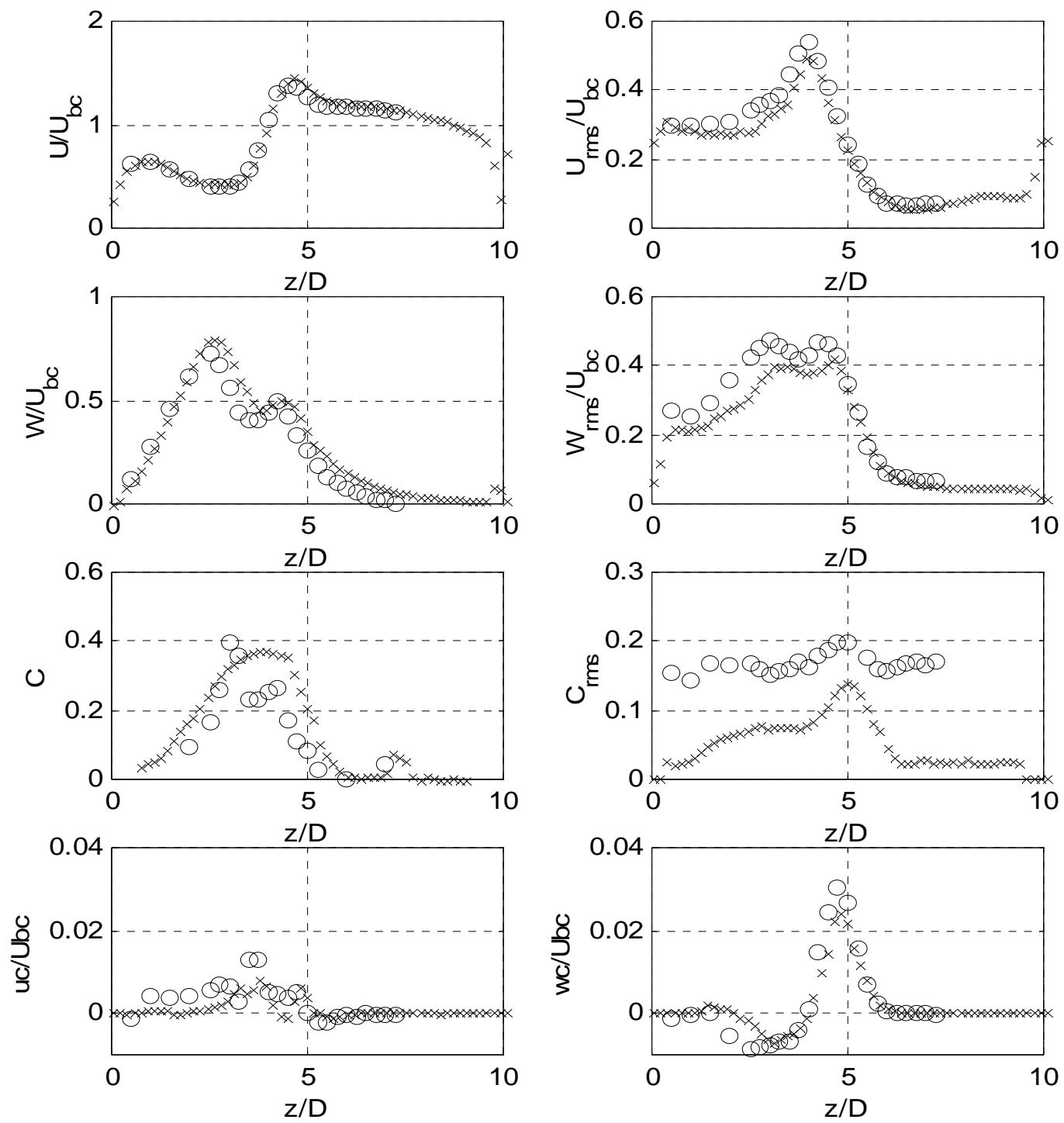

Fig. 8. Profiles of velocity components and concentration (mean and RMS values) for turbulent flow along a line through $\mathrm{x}=3 \mathrm{D}$. Symbols used in the plots: LDA/LIF: $\mathrm{O}$ and PIV/PLIF: $\times$.

\section{CONCLUSION}

Measurements by PIV/PLIF and LDA/LIF are presented for a jet in cross flow in a square duct. Measurements of the upstream velocity and Reynolds stresses show that data from both LDA and PIV have satisfactory accuracy and that the flow data are in good agreement with data from other workers. Detailed measurements of mean velocities, concentration and Reynolds fluxes are presented for a turbulent flow case. Similar measurements of mean velocity and concentration for a laminar flow case reveal differences between the laminar and turbulent flow cases. Dispite a choice of challenging cross sections with steep gradients the two techniques in general give results in good agreement. However, in a region with intense mixing just downstream of the jet trajectory some deviations are found. These deviations are mainly caused by the measuring volume of PIV/PLIF being too large to capture smaller scales in regions of high turbulence and mixing. 
The accuracy of the PLIF data (with summation of $8 \times 8$ pixel areas) based on measurements in uniform concentration is estimated to be $2.5 \%$. This level is for concentration levels found from. The 12-bit resolution for pixels is therefore more than adequate for concentration measurements. The final accuracy of the PIV/PLIF measurements are limited by having a large measuring volume relative to the jet diameter. Even though measurements of Reynolds fluxes probably show the correct distribution, their magnitudes are probably underestimated due to the size of measuring volume. More accurate measurements could be obtained by zooming into details of the flow, especially in the region near $(\mathrm{x}, \mathrm{z})=(3 \mathrm{D}, 3 \mathrm{D})$ of Figs. 7 and 8.

The accuracy of the LDA/LIF measurements based on measurements in uniform concentration is quite high with RMS values of $10-15 \%$. It is demonstrated that it is possible to make satisfactory measurements of the mean concentration and Reynolds fluxes in a relatively complex flow with a modified LDA system. However, accurate measurements of RMS values of concentration were difficult to perform due to high noise level in the LIF signal which proved to depend significantly on upstream concentration of Rhodamine.

\section{ACKNOWLEDGEMENTS}

The second author acknowledges the receipt of a grant in scope of the NATO Science Fellowship Programme by the Scientific and Technical Research Council of Turkey. He expresses his gratitude to Prof. P.S.Larsen for giving him the privilege to work at the Department of Energy Engineering of the Technical University of Denmark.

\section{REFERENCES}

Camussi, R., Guj, G. and Leone, A. "Experimental Analysis of Jets in Cross-Flow at low and moderate Reynolds number”, $8^{\text {th }}$ International Symposium on Flow Visualization, Sorrento, Italy, September 1-4, 1998.

Cenedese, A. and De Angelis , G. "Near Field Analysis of a Jet in Cross-Flow by means of LIF and PTV”, $8^{\text {th }}$ International Symposium on Flow Visualization, Sorrento, Italy, September 1-4, 1998.

Choi, Y. D., Iacovides, H. and Launder, B. E., “Numerical computation of turbulent flow in a square-sectioned 180 deg. bend”, Journal of Fluids Engineering, vol. 111, no. 1, p. 59, 1989.

Comte-Bellot,G., “Ecoulement turbulent entre deux parois paralleles” Publications Scientifiques et Techniques du Ministere de l'Air no.419, 1965.

Gavrilakis, S.,”Numerical simulation of low Reynolds number turbulent flow through a straight square duct”, Journal of Fluid Mechanics, Vol. 244, pp.101-129, 1992.

Houchine, I., Vivier, H., Plasari, R., David, R. and Villermaux, J., "Planar laser induced fluorescence technique for measurements of concentration fields in continuous stirred tank reactors”, Experiments in Fluids, vol. 32, pp. 95-102, 1996.

Jones, O. C., “An improvement in the calculation of turbulent friction in rectangular ducts”, Journal of Fluids Engineering, vol. 98, pp. 173-181, 1976.

Karasso, P. S. and Mungal, M. G., "PLIF measurements in aqueous flows using the Nd:YAG laser”, Experiments in Fluids, vol. 23, pp. 382-387, 1997.

Lima, M.M.C.L., Palma, J.M.L.M. and Silva, N.A., "Simultaneous Velocity and Concentration Measurements in Confined Coaxial Jets”, 8th International Symposium on Applications of Laser Anemometry to Fluid Mechanics, Lisboa, Portugal, Article N. 30.4, 8-11 July, 1996.

Owen, F. K., "Simultaneous Laser Measurements of Instantaneous Velocity and Concentration in Turbulent Mixing Flows”, AGARD CP 193, Applications of Non-intrusive Instrumentation in Fluid Flow Research, Saint-Lois, France, 35 May, 1976.

Scarano, F. and Riethmuller, M.L., Iterative multigrid approach in PIV image processing with discrete window offset, Experiments in Fluids, vol. 26, pp 513-523, 1999. 
Speiser, S. and Shakour, N. Photoquenching parameter for commonly used laser dyes, Appl. Phys. B, 38, pp 191197,1985

Ullum, U., Imaging techniques for planar velocity and concentration measurements, Ph.D. thesis, Department of Energy Engineering, Technical University of Denmark, 1999.

Yuan, L.L. and Street, R.L., Trajectory and entrainment of a round jet in crossflow, Physics of Fluids, Vol.10, No. 9, 1998. 